\title{
Reflective writing followed by dialogue improves supervision practices and cooperation in midwifery education
}

Anne-Lise Thoresen (Associate Professor) and Bente Norbye (Professor)

Faculty of Health Sciences/ Department of Health and Care Sciences/ UiT The Arctic University of Norway

\begin{abstract}
Writing as a tool for learning and reflection is acknowledged as important in education. This article discusses how writing and subsequent dialogue can support midwife supervisors in enhancing their supervisory skills. The aim of this article is to discuss the significance of reflective journals as a tool for reflection, dialogue and cooperation in supervision practices in midwifery education. An action research approach was used in six projects during 20082015, structured as a series of university based meetings interspersed with supervision of midwifery students in placement. The data consisted of reflective journals, which were systematically analysed in dialogue and cooperation with the participants. The data were further analysed, themed and interpreted using Ricoeur's philosophy of text analysis. Four themes emerged; 1 . A new, expanded understanding of supervision as a concept and practice. 2. A focus on the midwifery student as the learner. 3 . Awareness of the role of the supervisor. 4. The importance of student-supervisor cooperation. The process of systematic writing and subsequent dialogue enhanced the midwives learning and contributed to the comprehensive body of knowledge, i.e. the integration of practical, theoretical and supervision knowledge. New research will be important in further developing supervisory skills in midwifery education.
\end{abstract}

Keywords: Midwifery education, action research, supervision, reflective journal, dialogue

\section{Introduction}

This article will discuss how midwifery supervisors can be supported in their supervision work by using reflective writing as a basis for an elaborated dialogue. Reflective writing and the effectiveness of dialogue offer an approach to learning in supervision practices. While it is well established that reflection-on-action can enhance professional development (Schön 1983) and that writing can act as a core element in the process, it is important to explore how writing can transform reflection into a coherent and generative educational experience (see also 
Rowland 2000, 85-98). According to Wennergren and Rönnerman (2006), narrative writing can help practitioners explicate their experiences by acting as a means for reflective thinking. This is particularly appropriate for action research and practice-based research in general. Writing reflective journals can enable practitioners to gain better access to their own thoughts, to articulate and produce reflections, and to return to these reflections as the occasion arises (Wennergren and Rönnerman 2006). Francis (1995) sees reflective writing as a way for teachers to develop as reflective practitioners and to help them observe, analyse, reconstruct and deeply understand the process of personal theory building. Writing reflective journals can take the form of individual, collective and collaborative interactive processes, while sharing reflective journals can potentially contribute to teachers' professional growth (Cole et al. 1998; Wennergren and Rönnerman 2006).

Writing reflective journals can support reflection in different ways (Bolton 2010; Clandinin 2007; Collington and Hunt 2006), and may assist practitioners in developing reflective practices. According to Ralston (2005), reflective practices for midwives influence how midwives as practitioners approach and respond to situations and allow them to recall and think about their experiences to better understand their practices as midwives and student supervisors. Nakielski (2005) highlights reflection and reflective practice as important to develop professional midwifery practice and education. The activity of writing reflective journals can help midwife students, midwives working in clinical settings and midwife educators to better understand and improve professional midwifery and midwifery education. Reflective writing represents a convenient method for structuring reflection in a constructive and effective manner (Nakielski 2005). Dysthe, Hertzberg, and Løkengard Hoel (2010) emphasize writing as an important learning strategy and a way to manage one's own thoughts, where both thinking and writing are non-linear processes. This implies a continuous circular movement between what we think and write and the present moment. Writing safeguards our thoughts and allows us to recall them and follow their development. By returning to what has been written, we can unravel how our thoughts developed and discover new associations and trains of thought that we were unaware of in the moment they were written down. Such retrospective structuring is important to progress in developing our thinking and reasoning. Writing can help knowledge to become owned; knowledge is internalized as we question our own understanding. Writing and linguistic activity can activate the subconscious (Dysthe, Hertzberg, and Løkengard Hoel 2010). In developing enquiry of reflective practice in midwifery education, which includes professional supervision practice, writing reflective 
journals may provide an opportunity to link theory to practice and develop problem-solving skills, selfawareness and experience in critical thinking. Dialogue and collaborative learning may enhance individual reflection; when a learning environment fosters dialogue between students and teachers, space is created to approach learning in a new and innovative way (Brown et al. 2008)

The aim of this article is to discuss the significance of reflective journals as a tool for reflection, dialogue and cooperation in supervision practices in midwifery education. For the purpose of this research, reflective journals refer to documents written by midwife supervisors as part of learning strategies to systematically promote critical thinking to develop insight and engagement in supervision practices. The term 'supervisor' is not used consistently in the literature in Norway or in the United Kingdom and is frequently used alternately with other terms, such as 'mentor', 'guide', 'coach' or 'counsellor'. In this article, the term 'midwife supervisor’ refers to midwives supervising midwifery students in clinical practice.

The research question of this study is:

How can reflective writing and subsequent dialogue by midwives improve their ability to supervise midwifery students in clinical placements?

\section{Methodology}

\section{Context}

This article describes further research based on six action research projects developed by colleagues working in midwifery education at UiT The Arctic University of Norway. The projects were established as part of a local initiative aimed at supporting midwives in developing and refining their supervision practices for midwifery students in clinical placement. Promoting stronger collaboration between university-based midwifery educators and midwife supervisors was also important in enhancing the learning environment of midwifery students. Midwives working in clinical practice are responsible for the supervision of midwifery students but have little recourse to formal mentoring or any systematic form of supervision themselves.

A pilot project initiated in 2008 evaluated the need to further develop the midwives' supervision skills and provided direction for the subsequent projects. Following the pilot project, in the years 2009 to 2015, five independent projects were established and implemented. An average of 13 midwives took part in each project, including the pilot project. 
A total of 79 midwives participated in the action research projects from 2008 to 2015. Participants were all midwives recruited from maternity units under the Northern Norway Regional Health Authority, which covers the three northernmost counties, Finnmark, Nordland and Troms.

The projects were initiated locally with the aim of conducting research involving the development of midwives' supervision skills, a strengthening of collaboration and the acquisition of supervision knowledge to be used by midwives when supervising students. The participants were aware at an early stage that the data from the projects would be used empirically in research. In each of the projects, the midwives had an active participatory role that involved questioning their own and others' supervision practices, sharing experiences, producing new insights into supervision and reflecting on their own learning processes. In this way, the midwives could take control of their knowledge development, which, according to Carr and Kemmis (1986), can lead to insight and understanding. Further, McNiff $(2014,25)$ describes an active participatory role as involving 'a relational interest, about the need for dialogical relationships, where people talk together to improve their learning as the basis of improving their life-worlds'.

\section{Insider and outsider positions}

All the midwives who participated in the six projects from 2008 to 2015 wrote their individual reflective journals containing their reflections on their learning process. The reflective journals were shared among all the participants within each project, in a common journal. This common journal was included in the subsequence dialog and collaboration where challenges in the supervision of midwifery students were identified and discussed. In each of the six projects, two midwifery educators participated, being involved in the facilitation, management and implementation of the learning activities, as well as having responsibility for developing data to document the research process. As one of the two educators, the first author of this article participated as both educator and researcher in the six projects, which entailed a particular responsibility for the research. The effort to maintain openness about the role of the researcher and to provide a fair and transparent research process was an important requirement for the implementation of the six projects, as well as in the subsequent study discussed in this article. The researcher's position here can be called an insider position, where an educator is conducting research in collaboration with the other participants (Elden and Levin 1993, Herr and Anderson 2005). In the implementation of the six projects, the 
insider position meant that research work and learning activities were integrated in the collaboration with the other participants. In this collaboration, supervision knowledge, mutual understanding and insight into supervision were pursued and developed through dialogical processes. Such dialogical relationships are described as a window of opportunity for improved learning and development of the researcher's own knowledge (McNiff 2014).

This article describes a new perspective where a new external researcher, the second author, participated in the research. To ensure the credibility of the study, the second author contributed to the analysis of the data and the writing process. Creswell (2007) emphasizes the importance of researchers being aware of their subjectivity and recognizing the value-laden nature of research. In this research collaboration, we have aimed to combine the first author's unique access to and knowledge of the six projects with the more distanced role of the second author. This distanced role of the one researcher may be described as an outsider position. The insider/outsider positions thus enabled both proximity and distance to the original six projects, as well as analysis and interpretation of the data across the original projects. Both positions are valuable for the research processes and it is important to emphasize awareness of these positions in action research to ensure the quality of the study (Carr and Kemmis 1986; Elden and Levin 1993; Herr and Anderson 2005; McNiff 2014).

\section{Theoretical framework}

The theoretical framework for this study is linked to sociocultural theories. Lave and Wenger (1991) emphasize that learning is primarily a social phenomenon, where we learn from participating in communities of learning or communities of practice. Learning is situated, i.e. determined by the context, which in this study is the development of supervisory skills in midwifery education. The community of learning enables midwives to enhance their knowledge of supervision in interaction with the other participants. Vygotsky’s (1978) concept of the zone of proximal development demonstrates the importance of cooperation with others in broadening the understanding of a person aiming to learn at a higher level. To support this learning process, different linguistic and cultural tools may be used to promote understanding. In this study, the writing of a reflective journal as a structured learning activity is an example of a mediated learning activity that is both individual and social. In the writing processes, the writer is in a continuous dialogue that extends forwards and backwards in time (Bakhtin 1998). In an interaction perspective, meaning may arise between participants, 
because each utterance introduces elements from the past into new dialogues and can create meaningful learning processes (Bakhtin 1981).

\section{Action research approach}

An action research approach was used; this is well-known as a methodology with a potential for developing and improving professional knowledge in global and local settings, and as a research approach that enables practitioners to cooperate, develop and make changes in their professional practice (Choucri 2005; Deery and Hughes 2004). Reflection and reflective practice are key aspects of action research and are recognized as appropriate for professional development and support in midwifery practices (Ralston 2005). Action research is also a strategy for data collection and analysis, using an iterative and reflective process (McTaggart 1994).

In this study, the 'moments of action research' model (Carr and Kemmis 1986) was adopted, involving the four cyclical stages of 1) planning learning strategies to improve supervision practice, 2) taking action by implementing the learning strategies, 3) observing and systematically documenting the intervention, and 4) reflection to give meaning to the data collected, while also ensuring the opportunity for dialogue among all participants. To achieve collaboration through dialogue among all participants, the learning processes were structured as a series of universitybased meetings interspersed with periods of supervision of midwifery students in clinical practice.

During the university-based meetings, a range of strategies were established, including reflective teams in the form of group supervision, lectures, seminars and reading groups. The learning strategies aimed to promote professional supervision knowledge by integrating theoretical knowledge (supervision theories, concepts and models), supervision practices and ethical knowledge in the learning and reflection process. To develop supervision and reflective skills, learning strategies were based on participants’ practicebased experiences during supervision sessions with midwifery students in periods between the university-based meetings.

Writing reflective journals, subsequent dialogue and data collection

In this study, writing and subsequent dialogue as a structured learning activity was important to foster professional development and supervision knowledge (Connelly and Clandinin 1990) and were also part of the systematic documentation of the intervention. Writing followed by 
dialogue was an active process which included reflection, argumentation and active participation in learning. As a record of activities at the end of each day, participants wrote a reflective entry by hand in their journals, inspired by the questions: What have you learned? How do you intend to use this knowledge/experience in your supervision of student midwives?

Reflective journals constitute the data in this study and were identified as a significant data source, using the four criteria of Ricoeur (1977). This way of understanding and identifying reflective journals as significant data was an important part of the research process. The criteria for identifying significant data were 1) experiences were expressed in words in reflective journals, 2) they were communicated to participating midwives, 3) they represented reality rather than fiction, and 4) they were included in and transformed into a (new) narrative, i.e. this study. The data from action enquiry were understood as relating to narrative and hermeneutical research as highlighted by Pushor and Clandinin (2009).

Data were gathered from reflective journals and developed throughout the six projects from 2008 to 2015. A total of 474 individual reflective journal entries were produced during the six projects. Individual journals were transcribed and incorporated into a Microsoft Word document, forming one common journal. Thirty-six common journals formed the basis for the research and analysis in this study.

As part of the action research cycles, the common journal was sent by email to the participants after each session and before the next meeting to involve them all in a reflective practice concerning the common text. The midwives' subsequent reflections on the common text led to dialogue and shared supervision experience during the next meeting. This dialogue also became a form of quality assurance of the action research process, which Lincoln and Guba (1985) called 'memberchecking', and helped to ensure the trustworthiness of the research.

\section{Ethical considerations}

The International Confederation of Midwives (ICM) has formulated an International Code of Ethics for Midwives (2014) that focuses on the nature and conduct of midwives' education, practice and research. Engaging with this literature emphasized the importance of continual dialogue among all participants regarding ethical issues and potential challenges.

The local branch of the Norwegian Centre for Research Data (NSD) at the university was approached in writing regarding reporting requirements, but replied that the project did not 
need to be reported to them as no data would identify any persons directly or indirectly. However, it was important to provide information about how data would be used; therefore, all participants were asked to give written consent to participate in the project, and this consent was provided. To protect participants' identities, we ensured that the authors of all the reflective journals would remain anonymous. Written information about the project was sent to each participant and the selection criterion was that the midwife supervised a midwifery student during the project period.

\section{Analysis}

The analysis process started with the initial project and developed continuously during the six subsequent independent projects. The data were analysed in an active and systematic process in collaboration with the participants throughout the projects, as shown in steps 1, 2 and 3 in Figure 1 below. Steps 4 and 5 took place after the projects to provide deeper insight into the data in order to interpret, thematize and theorize in response to the research question. To provide an overview of this complex analysis process, the model below highlights the analytical steps that build upon the prior analysis, as is well-known in action research circles (Carr and Kemmis 1986).

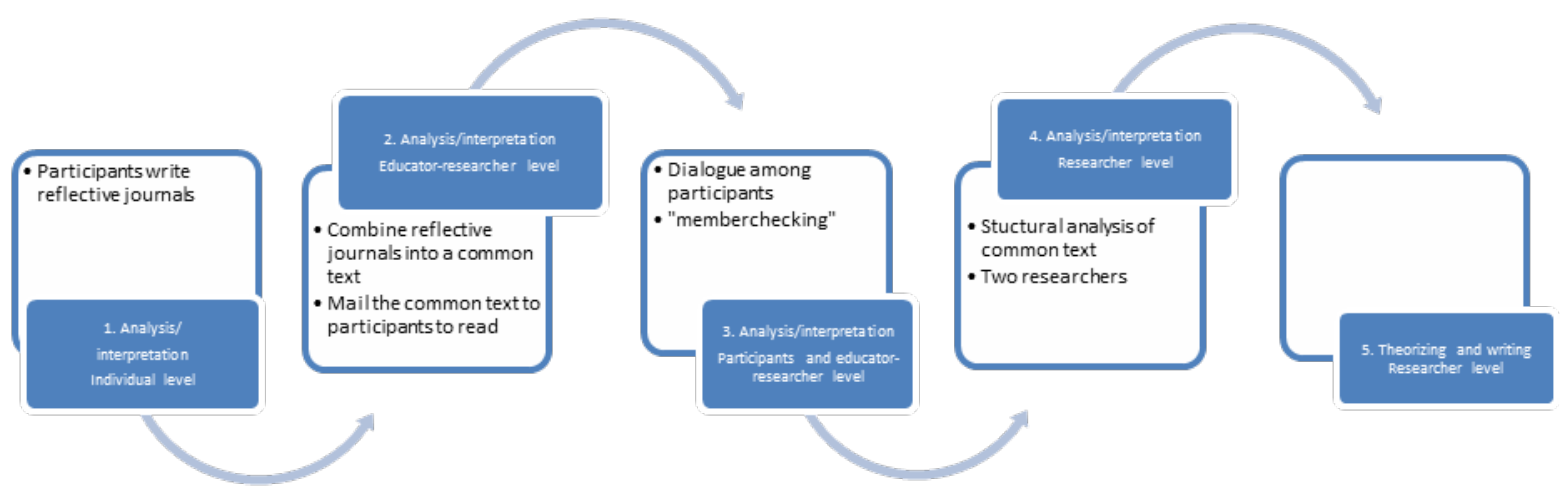

Figure 1. Five-step model of the analysis process.

Step one: Analysis/interpretation at the individual participant level; participants wrote individual reflective journals by hand.

Step two: Analysis/interpretation at the educator-researcher level. Reading individual written journals and transcribing each of them to create a common text in a Word document. 
Step three: Analysis/interpretation at the participant and educator-researcher level. Dialogue among participants.

Step four: Analysis/interpretation at the researcher level

This part of the analysis attempted to understand participants' experiences of writing reflective journals with subsequent dialogue as learning processes. Ricoeur’s (1976) philosophy of textual analysis as a phenomenological hermeneutical approach to researching narratives and lived experiences was used (see Ricoeur in Lindseth and Nordberg 2004). This served as a dynamic dialectical method that linked understanding and explanation, as illustrated in Figure 2. By reading the text, a deeper understanding was achieved; the text represented a broad spectrum of background conditions, including data, theory and experience. The experience of conducting action research with the midwives and researchers in a real-life context also served to further understanding.

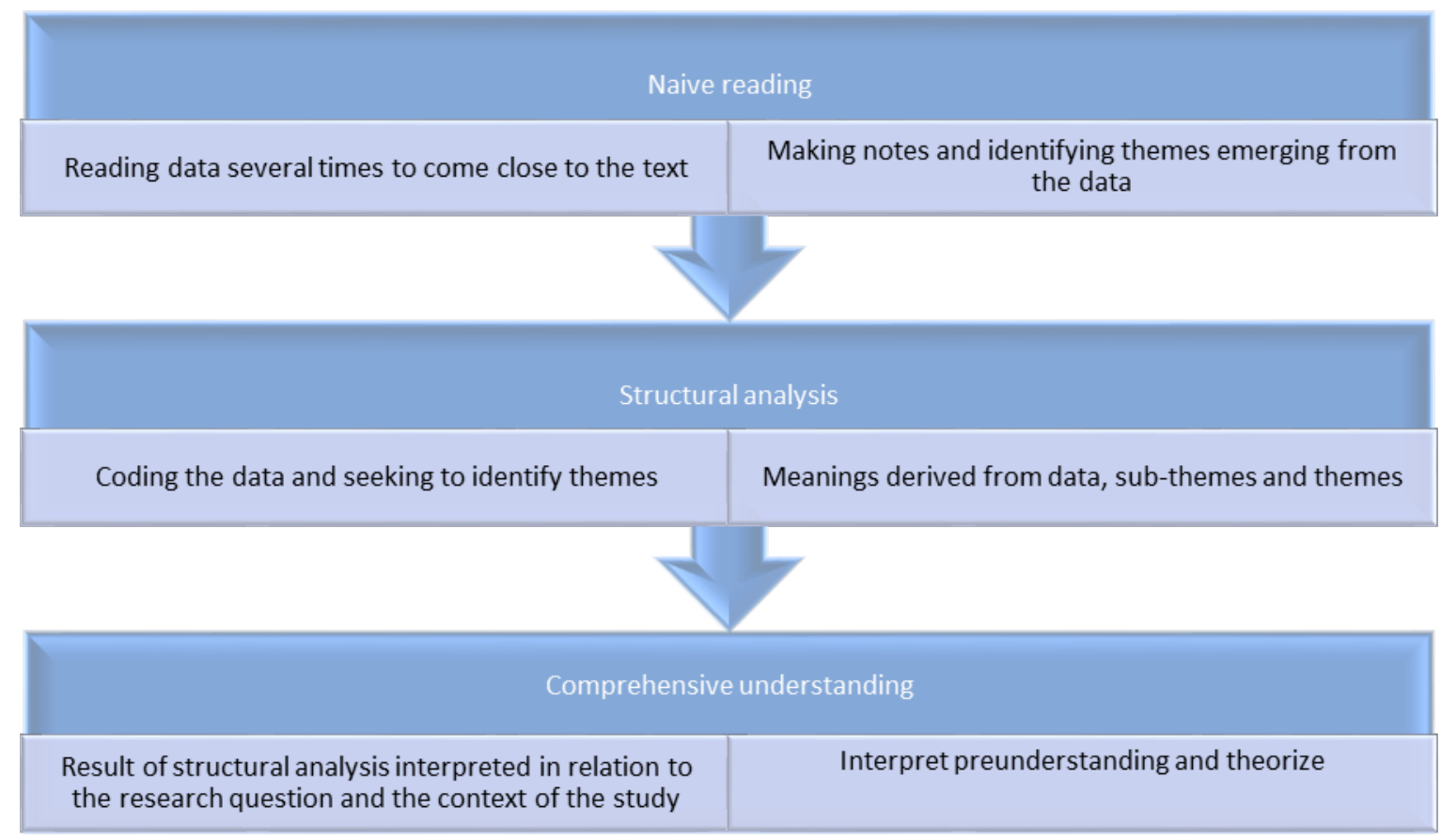

Figure 2. Model of Analysis/Interpretation.

Step five: Theorizing and writing - researcher level

Formulating the results of this study in a new narrative, as represented by and communicated through this article, involved drawing on insights from the other researchers and midwives, who acted as critical supporters throughout this writing project, providing input on its potential significance. However, in this part of the analysis, the position of an outsider researcher was adopted (McNiff 2014; Herr and Anderson 2005). To gain an outsider perspective, an external researcher (second author) who did not work in midwifery education 
but in the same department received professional support, and this cooperation provided distance to consider new perspectives on this research.

\section{Results}

The findings, following the analysis process, will reflect the research question: How can reflective writing and subsequent dialogue among midwifery supervisors improve their ability to supervise midwifery students in clinical studies?

The result of a structural analysis of written reflective journals in this learning context was formulated in four themes: 1) A new, expanded understanding of supervision as a concept and practice, 2) A focus on student learning, 3) Awareness of the role of the supervisor, and 4) The importance of student-supervisor cooperation.

A new, expanded understanding of supervision as a concept and practice

The learning strategies enabled participants to develop knowledge, insight and experience of supervision, which they could integrate into their professional competence as midwives. Systematic critical reflection provided them with the opportunity to gain insight into theoretical knowledge and supervision concepts, and into their own learning and understanding. Experience of reflection processes can enhance understanding of the concept of learning. The midwives appear to have developed their own action competence in encounters with students, including reflections on how students can learn and understand more in practice situations and on various aspects of their understanding of the role of a supervisor:

I think I can develop a clearer understanding of my own actions as a supervisor and my capacity to share knowledge with a midwife student. (Midwife Project 2015)

The responsibility of midwives as supervisors also became more visible:

I've become more aware of the responsibility and importance of planning the talk with the student -clarifying goals, wishes, learning needs and finding out where the student stands. I've realized the importance of being available, supervising as needed and giving students responsibility. (Midwife Project 2014)

The learning process involved a development and a new understanding: 
I've read and discussed articles that have taught me more about the role of the supervisor. I find I'm becoming more mature as a supervisor and I can find tools that can support me when I talk to students. (Midwife Project 2012)

An expanded understanding of supervision will affect the way midwives relate to students with greater scope for action in supervision situations. By including different learning perspectives, midwives could adopt different approaches to student learning during supervision and understand the importance of supervision as a systematic process that could be integrated into their own midwife practice.

\section{A focus on student learning}

The learning activities led to a stronger focus on the students' learning. During the projects, the midwives supervised students in clinical practice between the meetings at the university, thus gaining experience of supervision practices and the opportunity to reflect on this experience with the other participants. These midwives were able to understand and put words to their learning processes and to integrate this learning into supervision situations with students. Experiencing and articulating their own learning and knowledge development also facilitated their understanding of the students' learning.

I don't have all the answers but I can help the student to reflect and find the answers herself. (Midwife Project 2015)

Taking a step back as a supervisor was also important:

The student has to reflect personally on her own experiences; as a supervisor, I cannot impose my experiences on her. (Midwife Project 2012)

This midwife has discovered the importance of starting with the student's point of view. She demonstrates an expanded understanding of the reflection process and gives the student time to develop her own understanding.

The reflective team was useful. I learned to listen in a new way and to be aware of asking open questions. (Midwife Project 2010)

The time she needed for her own reflections was useful and she saw how it could be used for her students.

Using my experience as a method when I'm in dialogue with a student can help to empower the student and allow her to discover the answers for herself. (Midwife Project 2012) 
An important aspect of supervision practice is to gain experience of the importance of dialogue and the kind of questions to ask to enable students to learn and reflect on their actions. The midwives linked this experience to student learning and development. They learned the importance of developing a broader approach to student learning where supervision is a key tool to promote learning.

\section{Awareness of the role of a midwifery supervisor}

Awareness was a term that appeared in several of the participants' written journals; it may show that they have gained an understanding of concepts, events and experiences, and in this way midwifery supervision appears more evident to them, as the quotation below illustrates.

I became more aware of myself and my role as a supervisor for the midwife student. I want the student to be a good midwife, and I hope she will develop into a valued midwife colleague in the future. I want to adapt my supervision to my student's needs and give her an opportunity to reflect on her own knowledge and actions, motivation and understanding. (Midwife Project 2012)

The midwife's behaviour when meeting students was now seen as more important:

I've become more aware of the role of supervisor. I've become more aware of how I behave and relate to students and see their need for learning and practice. Hearing how the other midwives work with the students has given me insight into their experience. I listen more now and I think I ask better questions. I feel more confident now I have more knowledge about supervision. (Midwife Project 2014)

These quotes show that the midwives express how their supervision will contribute to the student's development into a competent midwife. The final quote contains a wish to meet students where they are and provide opportunities for reflection. This midwife has developed awareness of herself as a midwife and supervisor where she includes the student in her own practice. She has realized that supervision encompasses both values and clinical skills, and that all available knowledge is included in a situation. She has developed awareness of the student's dignity and wishes to guide the student to think for herself. Awareness can be a first step towards critical reflection, where the midwife understands and reflects on the importance of the student's learning and supervision needs. 


\section{The importance of cooperation}

Cooperation and dialogue were key aspects of learning in the projects. Knowledge and insight into communication and cooperation in midwifery are emphasized as important qualities of the professional midwife. It was important for the participants to experience how their written and oral reflections enhanced their understanding of their supervision practice. In dialogue with the other participants, this experience could be developed and used to improve understanding of student learning processes. When supervision is a collaborative effort between midwife and student, an equal relationship can arise where they both complement each other's knowledge even though they have different roles:

I've come to realize that supervision means collaboration between me and the student. I must be open and listen - and not lecture her - so that the student gets to talk about her experiences and reactions. In that way, I can reflect with her. (Midwife Project 2010)

This quote refers to a reflection where the participant has realized the importance of collaboration and sharing knowledge with the student to enhance learning and understanding. The supervisor listens to the student to gain insight into her understanding of a situation and bases further supervision on this communication. For the midwife, awareness of the cooperation aspect of supervision will lead to new opportunities and perspectives in the development of supervisory and communication skills.

\section{Discussion}

It is difficult if not impossible to state categorically that reflective writing and dialogue by midwifery supervisors improved their ability to supervise midwifery students. However, the projects altered their knowledge and insights into how they would supervise future students, how students could be part of a team and how students could be encouraged to develop and think for themselves as a learning process. The supervisors' own learning processes contributed to these deeper insights and self-understanding. The dialogue and collaboration enabled enhanced reflection and interpretation of the common journal and promoted the integration of theory and practice, which could then be included in the midwives' everyday supervision practice. This is consistent with Schön’s reflection-onaction $(1983,1987)$; the midwives considered their own experiences, and drew on theories to use in their supervision practice. This deeper insight entails building new understandings to inform new situations that unfold. 
As noted above, the theoretical framework is based on sociocultural theory, action research and a narrative approach. In this research project, oral and written dialogues were the most important and basic tools. The dialogues were conducted in small and larger groups through conversational interaction and by reading articles by key authors. Speech has a central position in sociocultural theory, while according to Vygotsky (1987), writing is an important medium for thinking and learning processes. Through writing, our thoughts can be tracked, discovered and developed. This concurs with Wennergren and Rönnerman (2006) emphasis on writing as an important reflection tool that can promote inner dialogue and lead to deeper insight and knowledge. This retrospective activity is important for maintaining reflection and a train of thought, which are also important aspects of an action research cycle. According to Bakhtin (1981), understanding and developing greater insights are active processes, and responses from other participants are vital. When participants discussed the transcripts of their reflective journals, which were now in the form of a common reflective journal, new meaning was created through the dialogical interaction. This reflection process - writing and dialogue, in light of the concept of the zone of proximal development, will enhance insight into supervision and personal knowledge, as Wennergren and Rönnerman (2006) highlight in their study. In our study, we see that both inner dialogue and reflection were developed in the writing process and especially clearly through the group dialogue; this can be understood as a meaning-creating process that changed the participants' knowledge of supervision. The dialogic interaction in the group enabled a discussion of experiences based on a common text, aiming to reach a mutual understanding of supervision. Here, both concrete supervision experience and theoretical knowledge were important for reflection and improved supervision practice with a clearer focus on the students.

Reflective writing, as part of a process of systematic reflection, provides an opportunity for 'reflection-on-action', to reflect on, re-live and re-experience learning and understanding (Attard 2012) as a tool for reflective practice. In this action research perspective, this multilayered concept could be realized by developing cultures of reflective enquiry. In this way, narrative writing became a form of inquiry, reflection and observation that was inseparable from the action research process itself. The participating midwives found that reflection was essential for developing professional midwifery and supervision practices, and that reflection may be accomplished in a systematic way, as in the context of writing reflective journals. The participants saw how holding back their own knowledge and giving students time to reflect for themselves would benefit student learning. As argued by Nakielski (2005), writing 
reflective journals encouraged reflection-on-action among the midwives and enabled them to analyse individual supervision experiences. When each participant wrote about what she had learned, she focused on experiences from different learning activities during the course, such as practising supervision in reflective teams (as a form of group supervision), lectures, seminars, and reading groups.

Focusing on supervision experiences from a retrospective perspective may trigger an awareness of prospective understanding and promote reflection before- and in-action in accordance with supervision and midwifery practices. Following Carr and Kemmis (1986), 'the moments of action research', namely, writing reflective journals, helped the participants develop awareness of reflective practice as looking back as retrospective understanding, and looking forward as prospective understanding, to the next action. The following comment from a midwife's reflective journal may illustrate the self-reflecting spiral (Carr and Kemmis 1986) and awareness of being a midwifery student supervisor:

I am excited about how much we have learned when we talk about it with other participants, especially when I see it in copied form as a text. I can understand what we were doing and talking about much more clearly. (Midwife Project 2012)

This quote can be understood as communicating a retrospective and prospective dialogue with the self through writing, cf. Arendt's (1978) concept of thinking as dialogue with the self. The midwives seem to have developed an awareness of student supervision through their reflective thinking and writing, and they found that writing could help turn reflection into a more coherent and revisitable learning experience. Individual reflection is enhanced by dialogue in groups and collaboration. Dialogue and articulation to others may clarify and encourage the reconstruction of existing knowledge, as highlighted by Francis (1995).

The example in the quote also demonstrates how writing can strengthen a midwife's understanding through engagement in dialogue with herself while she is writing. From a sociocultural perspective, Bakhtin’s (1981) theory sheds light on how meaning and understanding take place; these are important elements for gaining insight into what is involved in the processes of learning and communication. Meaning is not created primarily in the text, but meaningful words are created in dialogue with other participants in the subsequent communication and in the context of learning activities with the other participants (Bakhtin 1981). 
According to Dysthe, Hertzberg, and Løkengard Hoel (2010), writing as part of a learning process is based on an assumption that it can be a way of becoming aware of thoughts, developing them and structuring them in order to clarify our thinking. Through this reflective process, we can discover the beginnings of new lines of thought of which we were unaware at the time the thoughts were written down. This structured retrospective process frequently contains an assumption that it is possible to go further in developing one's thoughts. Writing helps to focus our thoughts and thus achieve a higher level of abstraction (Dysthe, Hertzberg, and Løkengard Hoel 2010). As a writer, one often finds oneself in a continuous back-andforth conversation (see Bakhtin 1998). The combination of individual writing and the interaction and reciprocal dialogue promoted the learning potential, as described by Dysthe (2002), and enhanced the individual learning of each midwife. The dialogue and cooperation between midwives thus became meaningful, in terms of Bakhtin's (1981) statement that understanding is always created in dialogue between the speaker and the listener, and the writer and the reader.

Individual writing followed by conversation became an important part of learning and reflection strategies in our projects. However, according to Carr and Kemmis (1986), it is important to discuss actions (in this context, writing reflective journals) together with participation in an interaction context. From a learning and research perspective, it was important to look more closely at individual written reflective journals and make them accessible for dialogue and further reflection about supervision and midwifery practices among the participants. The idea of combining written reflective journals and dialogue among participants became a key aspect of the action research process.

Furthermore, speech is the most important means of communication and helps to organize our inner world (Vygotsky 1987). Retrospective structuring of thoughts is important for maintaining trains of rational thought within a world of thoughts. Writing can help to understand the interaction between individual and sociocultural factors (Cole 1985); in a research context, this implies the interaction between the processes of data gathering and analysis. Vygotsky's concept of the zone of proximal development can provide understanding of the relationship between individual writing and thinking and sociocultural interaction. Hence, individual thinking also created an awareness that having the students in placement could be developed into teamwork. In such a team, the midwife and student could jointly care for the woman in labour and complement each other; the student's new knowledge and a different perspective and way of thinking would supplement the experience of the midwife. 
The use of a narrative approach and data collection led to an understanding that writing reflective journals could be used to make the supervision experience explicit and encourage dialogue among participants, with the aim of constructing shared understandings and reciprocal knowledge about midwifery student supervision. It was important in this study to achieve a deeper understanding of action research and reflective journals. According to Nicholls and Webb (2006), having good communication skills is the most important attribute, or competence, of being a good midwife. Other major competences are being compassionate, supportive, knowledgeable, and skilful. Being a good supervisor for student midwives will influence student practices when working with pregnant women (Nakielski 2005). The reflective journals enhanced her awareness of her role as supervisor and her support for student learning.

\section{Conclusion}

The participants stated that the reflective practice acquired on the course had improved and altered their ability to supervise their students in placement. Further studies would have to verify that such courses actually alter supervision in practice, but we see that the reflective writing and subsequent dialogue enabled a new and deeper dialogue that the midwives discovered through the processes led by the university. This study may therefore serve as an original contribution to the literature of midwifery supervision practices and to the wider field of healthcare supervision.

Some implications for midwifery supervision practices

Our joint discussions were wide-ranging and involved the analysis and interpretation of dialogical processes, the nature of communication and supervision methods, pedagogical knowledge in supervision, the importance of empathy, and awareness of how reflection processes can help the individual gain self-knowledge in supervisory skills. In our view, the development of this capacity for reflection can transform into a metacompetence or an overarching competence through which one can position oneself outside the practice field and make judgements about the quality of one's own practices. This process of self-reflection can then amount to the development of practical wisdom, as expressed in Schön's (1983) concept of the reflective practitioner. In terms of this paper, we suggest that evolving into a reflective midwife with supervisory responsibility for midwifery students must include this kind of analytical ability and critical thinking. It may also include the ability to develop oneself, to 
make wise and informed judgements and to consider ethical implications. This constitutes a comprehensive body of knowledge that includes practical wisdom, i.e. the integration of practical, theoretical and personal midwife and supervision knowledge. Such practical wisdom ought to be seen in all pedagogical and counselling interactions with students. In our view, this high level of knowledge constitutes the kind of professional expertise for which all midwife supervisors must strive and which can act as the hallmark of a noble profession.

\section{Summary}

The article discusses a key focus that emerged in the research: supporting the midwife supervisors in keeping reflective journals, the findings of which could inform future practices of other midwife supervisors. In this study, cooperative cultures of enquiry appeared to facilitate the integration of different types of knowledge. This enabled practising midwives, in their roles as professional student supervisors, to support midwifery students more effectively by refining their own learning. The development of reflective cultures of enquiry has the potential to ensure that pregnant women have an optimal experience during childbirth. Data from the journals show that this integration of forms of knowing can give deep meaning to midwives’ work. Comments from the participants' journals reveal a growing awareness of themselves as professionals.

\section{Limitations of the study}

This study refers to context-specific projects where the data were developed over time through interactive processes and dialogue, and the findings are thus not generalizable or directly transferable to other contexts. This study cannot show that the projects have improved the midwives' ability to supervise their students, but is limited to the reflective process shown in the material. Further research using interviews and observation studies might lead to different findings.

The role of the first author as both facilitator and researcher with an insider position could have led to bias, emphasizing the positive outcomes of the findings. However, the limits of this research lay within this perspective and the authors take full responsibility for the interpretations in this research. 


\section{References}

Arendt, H. 1978. The Life of the Mind: One/Thinking. New York: Harcourt Brace Javanovich.

Attard, K. 2012. “The Role of Narrative Writing in Improving Professional Practice.” Educational Action Research 20 (1):161-175. doi:10.1080/09650792.2012.647754.

Bakhtin, M.M. 1981. The Dialogical Imagination, edited by C. Holquist, 269-360.Austin and London: University of Texas Press.

Bakhtin, M.M. 1998. Spørsmålet Om Talegenrane [The Problem of Speech Genres]. Translated with an epilogue by R. T. Slaattelid. Bergen: Ariadne Forlag.

Bolton, G. 2010. Reflective Practice: Writing and Professional Development. Los Angeles, CA: Sage.

Brown, S.T., M.K. Kirkpatrick, D. Mangum, and J. Avery. 2008. “A Review of Narrative Pedagogy Strategies to Transform Traditional Nursing Education.” Journal of Nursing Education 47 (6): 283-286.

Carr, W., and S. Kemmis. 1986. Becoming Critical: Education, Knowledge, and Action Research. London: Falmer Press.

Choucri, L. 2005. "Creating Change: Developing a Midwifery Action Research Project.” British Journal of Midwifery 13 (10): 628-632. doi:10.12968/bjom.2005.13.10.19837.

Clandinin, D.J. 2007. Handbook of Narrative Inquiry: Mapping a Methodology. Thousand Oaks, CA: Sage.

Cole, M. 1985. "The Zone of Proximal Development: Where Culture and Cognition Create Each Other.” In Culture, Communication and Cognition, edited by J. Wertsch, 146-161. Cambridge: Cambridge University Press.

Cole, R., L. Raffier, P. Rogan, and L. Schleicher. 1998. “Interactive Group Journals: Learning as a Dialogue among Learners.” TESOL Quarterly 32 (3): 556-568. doi:10.2307/3588126.

Collington, V., and S. Hunt. 2006. "Reflection in Midwifery Education and Practice: An Exploratory Analysis.” Evidence Based Midwifery 4 (3): 76-82.

Connelly, F.M., and D.J. Clandinin. 1990. "Stories of Experience and Narrative Inquiry.” Educational Researcher 19 (5): 2-14. doi:10.3102/0013189x0190050.

Creswell, J.W. 2007. Qualitative Inquiry and Research Design. Choosing among Five Traditions. 3rd ed. Thousand Oaks, CA: Sage Publications.

Deery, R., and D. Hughes. 2004. "Supporting Midwife-led Care through Action Research: A Tale of Mess, Muddle and Birth Balls.” Evidence Based Midwifery 2 (2): 52-58.

Dysthe, O. 2002. "The Learning Potential of a Web-mediated Discussion in a University Course.” Studies in Higher Education 27 (3): 339-352. doi:10.1080/03075070220000716.

Dysthe, O., F. Hertzberg, and T. Løkengard Hoel. 2010. Skrive for Å Lære. Skriving I Høyere Utdanning [Write to Learn. Writing in Higher Education]. Oslo: Abstrakt forlag AS. doi:10.1080/ 03075070220000716.

Elden, M., and M. Levin. 1993. "Cogenerative Learning. Bringing Participation into Action Research.” In Participatory Action Research, edited by W.F. Whyte, 127-142. Newbury Park, Calif.: Sage Publications.

Francis, D. 1995. “The Reflective Journal: A Window to Preservice Teachers’ Practical Knowledge.” Teaching and Teacher Education 11 (3): 229-241. doi:10.1016/0742051X(94)00031-Z. Herr, K., and G. Anderson. 2005. The Action Research Dissertation. New York: Sage. 
International Confederation of Midwives. 2014. Code of Ethics for Midwives. The Hague: Author. Accessed 9 Oct 2019. https://www.internationalmidwives.org/assets/files/generalfiles/2019/10/ eng-international-code-of-ethics-for-midwives.pdf

Lave, J., and E. Wenger. 1991. Situated Learning - Legitimate Peripheral Participation. Cambridge: Cambridge University Press.

Lincoln, Y.S., and E.G. Guba. 1985. Naturalistic Inquiry. Newbury Park, CA: Sage. Lindseth, A., and A. Nordberg. 2004. "A Phenomenological Hermeneutical Method for Researching Lived Experience.” Scandinavian Journal of Caring Sciences 18 (2): 145-153. doi:10.1111/j.14716712.2004.00258.x.

McNiff, J. 2014. Writing and Doing Action Research. London: Sage.

McTaggart, R. 1994. "Participatory Action Research: Issues in Theory and Practice.” Educational Action Research 2 (3): 313-337. doi:10.1080/0965079940020302.

Nakielski, K.P. 2005. “The Reflective Practitioner.” In Decision Making in Midwifery Practice, edited by M.D. Raynor, J.E. Marshall, and A. Sullivan, 143-156. Edinburgh: Elsevier Churchill Livingstone.

Nicholls, L., and C. Webb. 2006. "What Makes a Good Midwife? an Integrative Review of Methodologically-Diverse Research.” Journal of Advanced Nursing 56 (4): 414-429. doi:10.1111/ j.1365-2648.2006.04026.x.

Pushor, D., and D.J. Clandinin. 2009. "The Interconnections between Narrative Inquiry and Action Research.” In The SAGE Handbook of Educational Action Research, edited by S. Noffke and B. Somekh, 290-300. London: Sage.

Ralston, R. 2005. "Supervision of Midwifery: A Vehicle for Introducing Reflective Practice." British Journal of Midwifery 13 (12): 792-796. doi:10.12968/bjom.2005.13.12.20127.

Ricoeur, P. 1976. Interpretation Theory: Discourse and Surplus of Meaning. Fort Worth, TX: Texas University Press.

Ricoeur, P. 1977. “The Question of Proof in Freud’s Psychoanalytic Writings.” Journal of the American Psychoanalytic Association 25 (4): 835-871. doi:10.1177/000306517702500404. Rowland, S. 2000. The Enquiring University Teacher. Maidenhead: The Society for Research into Higher Education \& Open University Press.

Schön, D. 1987. Educating the Reflective Practitioner. Toward a New Design for Teaching and Learning in the Professions. San Francisco, CA: Jossey-Bass.

Schön, D.A. 1983. The Reflective Practitioner. How Professionals Think in Action. New York, NY: Basic Books.

Vygotsky, L.S. 1978. Mind in Society. Edited by M. Cole, V. John-Steiner, S. Schribner and E. Souberman. Cambridge, MA: Harvard University Press

Vygotsky, L.S. 1987. “Thinking and Speech.” In The Collected Works of L.S. Vygotsky, Vol. 1, Problems of General Psychology N. Minick, Trans, edited by R.W. Rieber and A.S. Carton, 39-285. New York: Plenum Press.

Wennergren, A.-C., and K. Rönnerman. 2006. "The Relation between Tools Used in Action Research and the Zone of Proximal Development.” Educational Action Research 14 (4): 547-568. doi:10.1080/09650790600975791. 